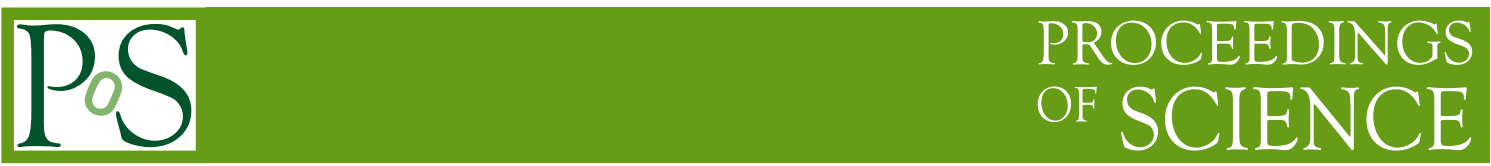

\title{
Holographic Superfluidity from a Magnetic Field
}

\author{
Yan-Yan Bu \\ Max-Planck-Institut für Physik (Werner-Heisenberg-Institut), \\ Förhringer Ring 6, 80805 München, Germany. \\ State Key Laboratory of Theoretical Physics, Institute of Theoretical Physics, Chinese Academy \\ of Science, Beijing 100190, People's Republic of China \\ E-mail: yybu@mpp.mpg.de \\ Jonathan P. Shock, Johanna Erdmenger and Migael Strydom* \\ Max-Planck-Institut für Physik (Werner-Heisenberg-Institut), \\ Förhringer Ring 6, 80805 München, Germany. \\ E-mail: (jke, jonshock, mstrydom) @mpp.mpg.de
}

Making use of gauge/gravity duality, we study two strongly coupled field theories that are the holographic duals of $S U$ (2) Einstein-Yang-Mills gravitational theories on asymptotically Anti-de Sitter backgrounds. One gravity setup is dual to a finite temperature field theory, while the other has a hard wall cutoff and is dual to a confining gauge theory. Both of these models undergo a superconducting phase transition at large enough values of the magnetic field. This yields a condensate in the field theory that has a ground state resembling Abrikosov vortices of a type II superconductor to linear order in perturbation theory. These models are related to p-wave superconductors where the condensate is induced by a finite isospin density. The results presented here may be of relevance to both condensed matter physics and heavy ion collisions. They may also provide support for recent proposals that the QCD vacuum may be unstable to the formation of a $\rho$ meson condensate when a strong magnetic field is present.

Xth Quark Confinement and the Hadron Spectrum

8-12 October 2012

TUM Campus Garching, Munich, Germany

${ }^{*}$ Speaker. 


\section{Introduction}

Gauge/gravity duality has been a remarkably successful tool for investigating the phase diagram of large $\mathrm{N}$ gauge theories. In the work [1], these investigations showed that an $S U(2)$ magnetic field can play the role of an order parameter. This order parameter triggers a superconducting phase transition in a strongly coupled field theory holographically dual to an $S U(2)$ Einstein-YangMills model on an $\mathrm{AdS}_{5}$-Schwarzschild background. Then, in [2], the ground state of the new phase was found. The ground state is, to linear order in an expansion of the condensate, equivalent to the triangular lattice of Abrikosov vortices from type II superconductors. At the conference "Xth Quark Confinement and the Hadron Spectrum" we were given the opportunity to present the findings of [2], which we review in these proceedings.

The model we present is a cousin of holographic p-wave superconductors where the condensation is induced by a finite isospin density. These are holographically realised by a non-trivial temporal component of the $S U(2)$ gauge field (see [3] and [4, 5] as well as the recent [6]). Our model is thus a simple extension: we switch on a nonzero spatial component of this gauge field instead of the temporal component. Whereas in $[4,5]$, a Meissner effect is shown to occur by which a magnetic field reduces the transition temperature, here it is the magnetic field which induces condensation at zero density.

The setup in these proceedings is interesting in the broader context of holographic lattices. In [7], the authors studied the holographic construction of an Einstein-Maxwell-scalar theory at finite temperature and density. They broke the translational invariance explicitly by imposing scalar field boundary conditions in the form of a lattice modulated in one of the Minkowski spatial directions. Other works have focused on lattices resulting from spontaneous breaking of translational symmetry. The translational symmetry can be broken by a Chern-Simons term, as shown in $[8,9,10,11,12,13]$, by a magnetic field, as shown in $[14,15]$, or by magnetic monopoles, as shown in $[16,17]$

Our holographic construction can also be seen as a model for a phenomenon first described by Chernodub et al. in $[18,19]$. There it was proposed that the $\mathrm{QCD} \times \mathrm{QED}$ vacuum may be susceptible to a superconducting phase transition when a magnetic field of the order of the QCD scale is present. This proposal came about through the study of a model proposed by Djukanovic, Schindler, Gegelia and Scherer in [20], which is an effective description of $\rho$ mesons interacting with an electromagnetic field. For a large enough external magnetic field, it was shown that there is an instability of the vacuum leading to a condensation of charged and neutral $\rho$ mesons. This breaks the $U(1)$ gauge symmetry and leads to a superconductor with the quark-antiquark pairs in the mesons acting as Cooper pairs. Moreover a solution was found in which the $\rho$ meson condensate forms an Abrikosov lattice made up of superconducting vortices [21]. There is evidence that such strong magnetic fields may be present at RHIC and the ATLAS experiment at CERN ([22, 23]), so it is interesting to ask if traces of this $\rho$ meson condensate could be detected.

\section{Holographic setup}

We study a very simple model of a strongly coupled quantum field theory in $(3+1)$-dimensions with a global $S U(2)$ symmetry. Its gravitational dual is an Einstein-Yang-Mills theory on an asymp- 
totically $\mathrm{AdS}_{5}$ geometry with an $S U(2)$ gauge field. The action is

$$
S=\int d^{5} x \sqrt{-g}\left\{\frac{1}{16 \pi G_{N}}\left(R+\frac{12}{L^{2}}\right)-\frac{1}{4 \hat{g}^{2}} \operatorname{tr}\left(F_{\mu v} F^{\mu v}\right)\right\},
$$

where $\hat{g}$ is the Yang-Mills coupling, $G_{N}$ is the $5 \mathrm{D}$ gravitational constant and $L$ is the $\mathrm{AdS}_{5}$ radius. $R$ and $F$ are the Ricci scalar and Yang-Mills field strength respectively.

We consider the probe approximation, where the Yang-Mills term is small compared to the Einstein-Hilbert term, so that the gauge fields do not backreact on the geometry. We thus choose a fixed 5-dimensional background metric, given by

$$
d s^{2}=\frac{L^{2}}{u^{2}}\left(-f(u) d t^{2}+d x^{2}+d y^{2}+d z^{2}+\frac{d u^{2}}{f(u)}\right),
$$

where the asymptotically AdS region is at $u \rightarrow 0$. We study two different models. The first is a finite temperature model where the background is AdS Schwarzschild, first proposed in [24]. In this case, $f(u)=1-\frac{u^{4}}{u_{H}^{4}}$, where $u_{H}$ is the location of the planar black hole horizon. The Hawking temperature of the black hole is $T=1 / \pi u_{H}$. The second model is the hard wall cutoff model, proposed in [25, 26], where $f(u)=1$ and the geometry terminates at a radial distance $u_{C}$. This model corresponds to a zero temperature theory $\left(u_{H}=\infty\right)$, but it still has a scale $u_{C}$ which corresponds to a confinement scale in the gauge theory. Without loss of generality we choose units where $u_{H}=1$ in the finite temperature theory and $u_{C}=1$ in the confining theory. Making this choice ensures that all physical quantities are dimensionless.

The $S U(2)$ gauge field has components $\boldsymbol{A}_{\mu}^{a}$, where $a=1 \ldots 3$ and $\mu=t, x, y, z, u$. We work in the gauge $\boldsymbol{A}_{u}^{a}=0$. Some of these components should be dual to the magnetic field and others to the condensate in the field theory. When the magnetic field is small and the system is in the normal phase, we want a field strength tensor with $F_{x y}^{3}=B$ and all other components zero. As shown in [1], when $B$ is larger than a critical value $B_{c}$ the system enters a new phase in which some of the vanishing components of $F_{\mu \nu}^{a}$ become nonzero. These new nonzero components correspond to the field theory condensate. We look for the new ground state at some value of $B$ infinitesimally above $B_{c}$. In this case the correction to the normal phase solution will be small and a perturbative analysis can be made in a parameter $\varepsilon \sim \frac{B-B_{c}}{B_{c}}$. We thus write an ansatz for the expansion in the form

$$
\begin{aligned}
\mathscr{E}_{x, y} & =\varepsilon E_{x, y}+\varepsilon^{3} e_{x, y}+\mathscr{O}\left(\varepsilon^{5}\right), \\
\boldsymbol{A}_{y}^{3} & =x B_{c}+\varepsilon^{2} a_{y}^{3}+\mathscr{O}\left(\varepsilon^{4}\right), \\
\boldsymbol{A}_{x}^{3} & =\varepsilon^{2} a_{x}^{3}+\mathscr{O}\left(\varepsilon^{4}\right) .
\end{aligned}
$$

and solve the equations order by order in $\varepsilon$. Here $\mathscr{E}$ and $\boldsymbol{A}$ denote the non-perturbative fields, with $\mathscr{E}_{\mu}=\boldsymbol{A}_{\mu}^{1}+i \boldsymbol{A}_{\mu}^{2} . E_{x, y}$ and $e_{x, y}$ are first and third order contributions to the condensate components, respectively, while $a_{x, y}^{3}$ are second order corrections to $\boldsymbol{A}_{x, y}^{3}$. Note that we neglect even powers of the condensate components and odd powers of the magnetic field components. It turns out that we can turn off the $t$ and $z$ dependence of each component of the gauge field and still have consistent equations. Turning off the $t$ dependence guarantees a static solution. Turning off the $z$ dependence, where the $z$ direction is parallel to the magnetic field, yields a lattice in the $x, y$-plane. The structure of the equations of motion ensure that we can do all of the above consistently. See [2] for further details. 
The $S U(2)$ gauge freedom in the choice of $\boldsymbol{A}$ needs to be handled carefully, as discussed in [1]. The idea is to have a model of a superconductor ${ }^{1}$, which means there should be a $U(1)$ symmetry that is broken spontaneously. The authors of [1] take the infinitesimal fluctuations $E_{x, y}$ and form linear combinations that transform covariantly in the fundamental of a $U(1)$ subgroup of $S U(2)$, and then fix the remaining gauge symmetry. All the calculations can be done without these $U(1)$ covariant fields, however, so we will not use them in these proceedings.

\section{Lattice solutions}

In solving the PDE's, we follow the strategy of [27, 28]. To linear order, the solution to the equations of motion is given by

$$
\begin{aligned}
& E_{y}=-i E_{x}, \\
& E_{x}=\sum_{n=-\infty}^{\infty} C_{n} e^{-i n k y-\frac{1}{2} B_{c}\left(x-\frac{n k}{B_{c}}\right)^{2}} U(u) .
\end{aligned}
$$

The solution for $E_{x}$ is a general linear combination of all possible lowest energy states - higher energy solutions have been neglected. The function $U(u)$ is determined numerically, as shown in [1], and solving it determines the value for $B_{c}$. This is shown in [2] to be $B_{c} \approx 5.1$ for the AdS Schwarzschild model $\left(f(u)=1-u^{4}\right)$ and $B_{c} \approx 5.8$ for the hard wall model $(f(u)=1)$.

The constants $C_{n}$ and $k$ are not fixed to linear order. Their values are determined by minimizing the ground state energy of the system, which needs to be calculated at fourth order. In order to make this problem manageable, we first need to restrict the values that the $C_{n}$ can take. We do this by making use of some symmetries. We can argue that, since nothing in the setup is explicitly breaking translational invariance in the $x, y$-directions, the solution should be a highly symmetric lattice. This implies that the coefficients $C_{n}$ must have the same magnitude $\left|C_{n}\right|$ and moreover be periodic in some integer $P$, that is, $C_{n}=C_{n+P}$.

In [27], Abrikosov first studied the simplest solution, a square lattice, which had $P=1$. Later Kleiner et al. in [29] generalised the analysis by looking at $P=2$, with $C_{1}= \pm i C_{0}= \pm i C$. This choice of coefficients specifies a general rhombic lattice, with the shape of the rhombus controlled by varying $k$. Figure 1 shows how this works. The lengths of the lattice cell are given by $L_{x}=2 k / B_{c}$ and $L_{y}=2 \pi / k$. A triangular lattice is then obtained by choosing $k=3^{\frac{1}{4}} \sqrt{\pi B_{c}}$, and a square lattice by choosing $k=\sqrt{\pi B_{c}}$. We follow the approach of Kleiner et al, which is to compute the energy density of the lattice for a range of values of the ratio $R=L_{x} / L_{y}=k^{2} / \pi B_{c}$. The energy is computed numerically from the analytic expressions we obtain at each order.

The energy is calculated using the holographic dictionary. For the AdS Schwarzschild model, we have a finite temperature so we can calculate the free energy using $\mathscr{F}=-T \ln \mathscr{Z}=-S_{c l}$, where $S_{c l}$ is the classical action on the gravity side. In the hard wall case, we are simply calculating the energy of the field configuration, which is defined in terms of the classical action in the same way. The total energy diverges, so we calculate its average $\Omega$ over a lattice cell. Also, since we are only interested in whether the energy of a particular superconducting solution is lower than that of the

\footnotetext{
${ }^{1}$ Note that this is actually a superfluid because the gauge symmetry is mapped to a global symmetry on the boundary.
} 


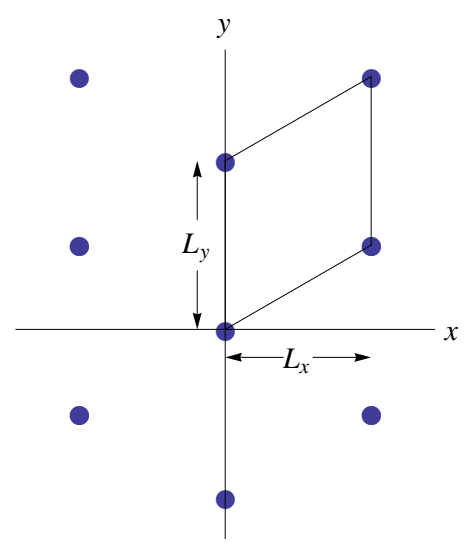

Figure 1: A lattice cell, illustrating the meanings of $L_{x}$ and $L_{y}$ for a fixed area cell.

normal phase solution, we can simply calculate the difference $\Delta \Omega=\Omega_{s}-\Omega_{n}$ and thus do not need to implement holographic renormalisation.

Solving the equations of motion to higher orders is technically challenging but not insightful, so it will not be done here. Many of the details are however found in [2].

\section{Results}

As explained above, the strategy is to fix values of $k, P, C_{n}=C_{n+P}$ and $B$ (close to $B_{c}$ ) then calculate the change in energy density $\Delta \Omega$. We only consider $P=2$ with $C_{1}=i C_{0}=i C$. Leaving $C$ general, we then substitute these values into the energy density. It takes the form $\Delta \Omega=a_{1} \varepsilon C+$ $a_{2} \varepsilon^{2} C^{2}+\ldots$. At this point we see that we can redefine $C$ by absorbing a factor of $\varepsilon$, which we call $C_{\varepsilon} . C_{\varepsilon}$ is the then only parameter left unfixed. Here the $a_{i}$ are values that are calculated numerically from substituting the solutions to the equations of motion into the expression for the energy. $\Delta \Omega$ forms a Mexican hat potential, which is easy to minimise numerically. The plot in figure 2 shows the energy-minimising value of $C_{\varepsilon}$ as a function of magnetic field near the phase transition at $B_{c}$. We see that $C_{\varepsilon} \sim\left(B-B_{C}\right)^{\frac{1}{2}}$, so the condensate ${ }^{2}$ has a critical exponent of $1 / 2$.

The curves in figure 3 show $\Delta \Omega$ for the square and triangular lattices. They are the result of calculations in the AdS Schwarzschild model, but we get the same results up to a rescaling of the axes for the hard wall model. Each curve shows that the free energy density is proportional to $\left(B-B_{c}\right)^{2}$. This shows that the phase transition is second order, as expected if one looks at the analogous case in Ginzburg-Landau theory. There one can show ([30]) that the free energy is proportional to $\left(T-T_{c}\right)^{2}$, where $T_{c}$ is the phase transition critical temperature.

The energy difference as a function of $R$ is plotted in figure 4. By looking at the lattice in figure 1, it is possible to see that the triangular lattice occurs for $R=L_{x} / L_{y}=\sqrt{3}$ and $R=1 / \sqrt{3}$. In general, $R$ and $1 / R$ give the same lattice but with the $x$ and $y$ directions flipped. This is why figure 4 displays the symmetry $\Delta \Omega(R)=\Delta \Omega(1 / R)$. The triangular lattice corresponds to a global minimum of the energy as a function of $R$, as seen from the figure. There is a local maximum for the square lattice, which is when $R=1$. As $R \rightarrow \infty$ (or $R \rightarrow 0$ ), the free energy increases.

\footnotetext{
${ }^{2}$ Note that only the combination $\varepsilon C$ is physically relevant, not $C$ or $\varepsilon$ independently.
} 


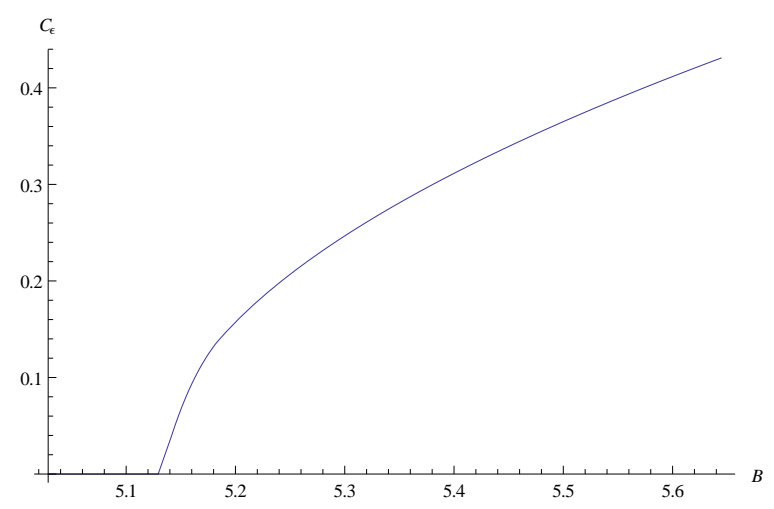

Figure 2: $C_{\varepsilon} \sim$ the overall condensate size for the AdS Schwarzschild solution in units of the temperature, as a function of the external magnetic field $B$. For $B<B_{c}$, the condensate is zero, and for $B$ slightly above $B_{c}$, we see a $\left(B-B_{c}\right)^{\frac{1}{2}}$ scaling behaviour. The plot for the hard wall model is the same, up to a scaling of the $B$ and $C_{\varepsilon}$ axes.

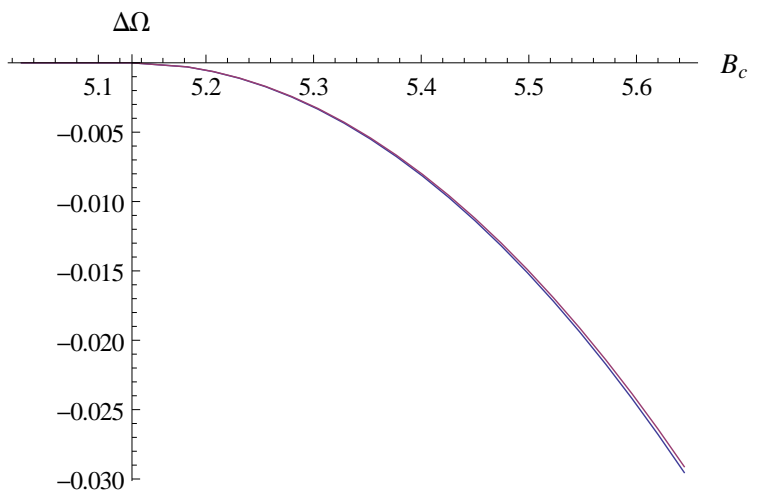

Figure 3: The change in energy density (compared to the normal phase) for the triangular and square lattices as the external applied magnetic field is varied. The phase transition happens at $B_{c} \approx 5.1$, which is where the coordinate axes are centred. $\Delta \Omega_{\text {square }}-\Delta \Omega_{\text {triangle }}$ is so small that the two plots are almost on top of each other. This is for the AdS Schwarzschild model, but the plots for the hard wall model are identical except for the scale on the axes. In the hard wall model, $B_{c} \approx 5.8$.

Intuitively one can understand this by making use of the properties of Abrikosov vortices that we understand from type II superconductors. These vortices repel. Since $R \rightarrow \infty$ and $R \rightarrow 0$ correspond to elongating the rhombic lattice cell (while keeping the area constant) neighbouring vortices are squeezed together, and since they repel, this is energetically unfavourable.

In figure 5 we present the contour plot of the modulus squared of the $x, y$-dependent factor of the linear order solution to $E_{x}$. It represents the minimum energy state corresponding to the triangular lattice. Using this solution to get the precise value of the condensate in the dual field theory is subtle, but it should take a similar triangular lattice shape. More details of how this is done can be found in [2].

\section{Conclusion}

In this work we have found a likely ground state for the Yang-Mills instability induced by 


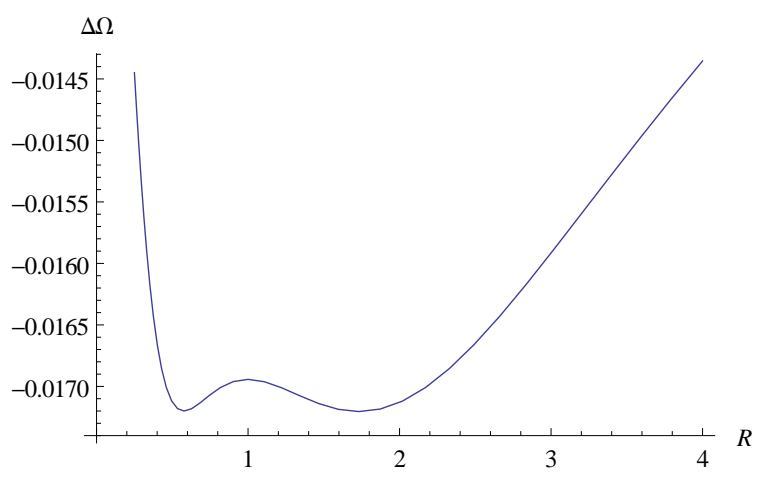

Figure 4: The change in free energy density as a function of $R=L_{x} / L_{y}$, the ratio of side lengths of a constant area lattice cell. This plot is for the AdS Schwarzschild model, but the plot for the hard wall model is identical up to a rescaling of the axes. When $R=1$, the lattice is square and the free energy achieves a local maximum. When $R=\sqrt{3}$ and $1 / \sqrt{3}$, the lattice is triangular and the free energy is at alobal minimum.

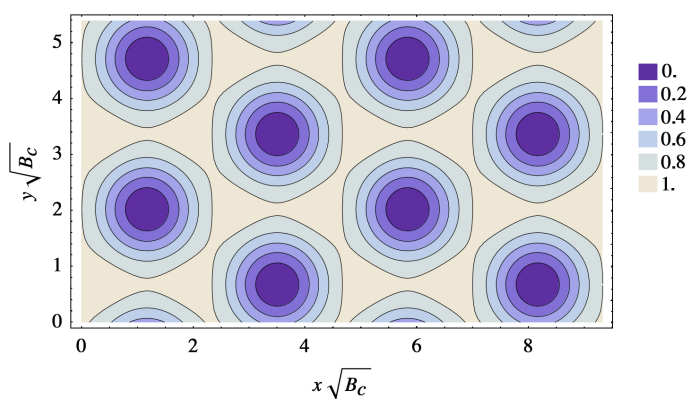

Figure 5: A contour plot of the modulus squared of the subleading term in the boundary expansion of $E_{x}$ for the ground state triangular lattice. The magnitude has been normalised to one. For the field theory interpretation of this result, see [2].

an $S U$ (2) magnetic field analysed in [1], as well as for a slightly different (hard wall) model. By looking at our model as an approximation of QCD, it provides supporting evidence for the claims of Chernodub et al. that the QCD vacuum with large magnetic fields is unstable to the creation of $\rho$ mesons. Being of a lattice form, it has much potential for analysis in condensed matter models as well, where the breaking of translational invariance has already been shown to be important in getting realistic phenomenology.

The two holographic models that we study have several important differences from QCD. In the finite temperature model there is no confinement or chiral symmetry breaking and so there are no goldstone bosons (pions) present, which are the normal decay modes of the $\rho$ meson in QCD. The hard wall model has its conformal symmetry broken only by an IR boundary condition which sets a confinement scale. However, the phenomenology of these two models appears to be close enough to that of QCD to compare qualitatively with the models of Chernodub et al.

In the present work we have analysed lattices with $P \leq 2$, corresponding to square and rhombic forms, respectively. Going to $P=3$ requires a large increase in computational power. While this would be an interesting further calculation, the analogous cases of type II superconductivity and the model of Chernodub et al. point to the triangular lattice being the true ground state. We thus 
expect higher $P$ lattices to be energetically disfavoured.

It is expected that if the QCD vacuum is unstable to $\rho$ meson condensation in extremely offcentre heavy ion collisions, then the timescale of the instability would not be enough to form a welldefined lattice. Abrikosov vortices may form, but the magnetic field would likely drop below the critical value before they had time to arrange themselves into a lattice. It would be very interesting to perform a real-time calculation in order study the formation of the vortices and their movements as the magnetic field increased and decreased through the lifetime of a single off-centre collision.

\section{Acknowledgments}

We are grateful to our collaborator Martin Ammon. M. S. and J. E. would like to thank the organizers of the conference "Xth Quark Confinement and the Hadron Spectrum" for a stimulating conference. M. S. would like to thank M. Chernodub for discussions. Y.Y.B. was supported by MPG-CAS Doctoral Promotion Programme. The work of J.S. was supported by the European Union through a Marie Curie Fellowship. The work of J.E. and M.S. was supported in part by the DFG cluster of excellence 'Origin and Structure of the Universe' (www.universe-cluster.de).

\section{References}

[1] M. Ammon, J. Erdmenger, P. Kerner and M. Strydom, "Black Hole Instability Induced by a Magnetic Field," Phys. Lett. B 706 (2011) 94 [arXiv:1106.4551 [hep-th]].

[2] Y. -Y. Bu, J. Erdmenger, J. P. Shock and M. Strydom, "Magnetic field induced lattice ground states from holography," arXiv:1210.6669 [hep-th].

[3] S. S. Gubser and S. S. Pufu, "The gravity dual of a p-wave superconductor," JHEP 11 (2008) 033, [arXiv:0805.2960].

[4] M. Ammon, J. Erdmenger, M. Kaminski, and P. Kerner, "Superconductivity from gauge/gravity duality with flavor", Phys. Lett. B680 (2009) 516-520, [arXiv:0810.2316].

[5] M. Ammon, J. Erdmenger, M. Kaminski, and P. Kerner, "Flavor Superconductivity from Gauge/Gravity Duality", JHEP 10 (2009) 067, [arXiv:0903.1864].

[6] S. Chunlen, K. Peeters, P. Vanichchapongjaroen and M. Zamaklar, "Instability of N=2 gauge theory in compact space with an isospin chemical potential," arXiv:1210.6188 [hep-th].

[7] G. T. Horowitz, J. E. Santos and D. Tong, "Optical Conductivity with Holographic Lattices," arXiv:1204.0519 [hep-th].

[8] S. K. Domokos and J. A. Harvey, "Baryon number-induced Chern-Simons couplings of vector and axial-vector mesons in holographic QCD,” Phys. Rev. Lett. 99 (2007) 141602 [arXiv:0704.1604 [hep-ph]].

[9] S. Nakamura, H. Ooguri and C. -S. Park, “Gravity Dual of Spatially Modulated Phase," Phys. Rev. D 81 (2010) 044018 [arXiv:0911.0679 [hep-th]].

[10] W. -y. Chuang, S. -H. Dai, S. Kawamoto, F. -L. Lin and C. -P. Yeh, "Dynamical Instability of Holographic QCD at Finite Density," Phys. Rev. D 83 (2011) 106003 [arXiv:1004.0162 [hep-th]].

[11] O. Bergman, N. Jokela, G. Lifschytz and M. Lippert, "Striped instability of a holographic Fermi-like liquid," JHEP 1110 (2011) 034 [arXiv:1106.3883 [hep-th]]. 
[12] C. A. B. Bayona, K. Peeters and M. Zamaklar, "A Non-homogeneous ground state of the low-temperature Sakai-Sugimoto model,” JHEP 1106 (2011) 092 [arXiv:1104.2291 [hep-th]].

[13] S. Takeuchi, "Modulated Instability in Five-Dimensional U(1) Charged AdS Black Hole with R**2-term,” JHEP 1201 (2012) 160 [arXiv:1108.2064 [hep-th]].

[14] A. Donos, J. P. Gauntlett and C. Pantelidou, "Spatially modulated instabilities of magnetic black branes,” JHEP 1201 (2012) 061 [arXiv:1109.0471 [hep-th]].

[15] A. Donos and J. P. Gauntlett, "Holographic striped phases," JHEP 1108 (2011) 140 [arXiv:1106.2004 [hep-th]].

[16] S. Bolognesi and D. Tong, "Monopoles and Holography,” JHEP 1101 (2011) 153 [arXiv:1010.4178 [hep-th]].

[17] P. Sutcliffe, JHEP 1108 (2011) 032 [arXiv:1104.1888 [hep-th]].

[18] M. N. Chernodub, “Superconductivity of QCD vacuum in strong magnetic field,” Phys. Rev. D 82 (2010) 085011 [arXiv:1008.1055 [hep-ph]].

[19] M. N. Chernodub, "Electromagnetically superconducting phase of QCD vacuum induced by strong magnetic field,” AIP Conf. Proc. 1343 (2011) 149 [arXiv:1011.2658 [hep-ph]].

[20] D. Djukanovic, M. R. Schindler, J. Gegelia and S. Scherer, "Quantum electrodynamics for vector mesons,” Phys. Rev. Lett. 95 (2005) 012001 [hep-ph/0505180].

[21] M. N. Chernodub, J. Van Doorsselaere and H. Verschelde, "Electromagnetically superconducting phase of vacuum in strong magnetic field: structure of superconductor and superfluid vortex lattices in the ground state,” Phys. Rev. D 85 (2012) 045002 [arXiv:1111.4401 [hep-ph]].

[22] V. Skokov, A. Y. .Illarionov and V. Toneev, "Estimate of the magnetic field strength in heavy-ion collisions,” Int. J. Mod. Phys. A 24 (2009) 5925 [arXiv:0907.1396 [nucl-th]].

[23] A. Bzdak and V. Skokov, "Event-by-event fluctuations of magnetic and electric fields in heavy ion collisions,” Phys. Lett. B 710 (2012) 171 [arXiv:1111.1949 [hep-ph]].

[24] E. Witten, “Anti-de Sitter space and holography,” Adv. Theor. Math. Phys. 2 (1998) 253 [hep-th/9802150].

[25] J. Erlich, E. Katz, D. T. Son and M. A. Stephanov, “QCD and a holographic model of hadrons," Phys. Rev. Lett. 95 (2005) 261602 [hep-ph/0501128].

[26] L. Da Rold and A. Pomarol, “Chiral symmetry breaking from five dimensional spaces,” Nucl. Phys. B 721 (2005) 79 [hep-ph/0501218].

[27] A. A. Abrikosov, "On the Magnetic properties of superconductors of the second group," Sov. Phys. JETP 5 (1957) 1174 [Zh. Eksp. Teor. Fiz. 32 (1957) 1442].

[28] A. A. Abrikosov, Fundamentals of the Theory of Metals, (North Holland, Amsterdam, 1988)

[29] W. H. Kleiner, L. M. Roth and S. H. Autler, "Bulk Solution of Ginzburg-Landau Equations for Type II Superconductors: Upper Critical Field Region,” Phys. Rev. 133 (1964) A1226-A1227 10.1103/PhysRev.133.A1226.

[30] M. Tinkham, Introduction to Superconductivity, (Robert E. Krieger Publishing Company, Malabar, Florida, 1980) 